\title{
Development of coated beads for oral controlled delivery of cefaclor: In vitro evaluation
}

\author{
BAZIGHA K. ABDUL RASOOL ${ }^{1}$ \\ SAHAR A. FAHMY2 \\ ${ }^{1}$ Department of Pharmaceutics \\ and Pharmacy Practice, Dubai Pharmacy \\ College, Dubai, UAE \\ 2 Department of Pharmaceutics \\ Faculty of Pharmacy, Helwan University \\ Helwan, Egypt
}

\begin{abstract}
The aim of the present study was to develop and characterize coated chitosan-alginate beads containing cefaclor as a controlled release delivery system. Coated cefaclor beads were prepared by solvent evaporation techniques. Beads were found to be intact and spherical in shape. Their size range was 1.05 to 2.06. The loading efficiency showed maximum value when the concentration of cefaclor, chitosan and PEG 400 was $10 \%(\mathrm{~m} / \mathrm{V}), 0.5 \%(\mathrm{~m} / \mathrm{V})$ and $2 \%(V / V)$, respectively. Best retardation of cefaclor release from chitosan-alginate beads was achieved by coating with $15 \%$ of shellac in formula F19. A significant antimicrobial activity $(p<0.05)$ against Staphylococcus aureus and Klebsiella pneumoniae was observed for formula F19 compared to the standard antibiotic disc. Furthermore, the simulated plasma profile showed the superiority of F19 in sustaining drug release for more than $12 \mathrm{~h}$. Therefore, shellac coated chitosan-alginate beads could be considered a successful controlled release oral cefaclor dosage form.

Keywords: cefaclor, chitosan-alginate, shellac coated beads, modified release, oral delivery
\end{abstract}

Development of sustained release dosage forms has attracted the attention of pharmaceutical manufacturers in recent years. Sustained release dosage forms can be formulated into a single unit dosage form such as tablet or capsule, or a multiparticulate dosage form such as pellet or bead. Recently, beads have attracted a considerable attention as potential drug carriers to obtain a controlled release, site-specific delivery and to increase drug bioavailability (1). Alginate and chitosan are the most widely used hydrogels in the formulation of such systems due to their high biodegradability and biocompatibility. Alginate is a natural polyacid and has a unique property of gel formation in the presence of multivalent cations such as calcium ions in aqueous media (2), while chitosan is a naturally occurring polysaccharide comprising glucosamine and $\mathrm{N}$-acetyl-glucosamine with unique polycation characteristics (3). Alginate beads have been reported in some studies for the problems during beads preparation. Drug loss and shrinkage of

\footnotetext{
*Correspondence; e-mail: bazigha_rasool@hotmail.com, bazigharasool@yahoo.com
} 
the alginate polymer take place when subjected to an acidic medium, while it dissolves at higher $\mathrm{pH}$ values (2). On the contary, chitosan beads dissolve at low $\mathrm{pH}$ and are insoluble in alkaline media. Some studies reported that chitosan alone cannot control drug release due to its hydrophilicity (3). Therefore, the alginate-chitosan polyelectrolyte complex was considered to be a promising medium to reduce the porosity of alginate beads and decrease the leakage of the encapsulated drug (4). Furthermore, one approach to obtaining more controlled release of drugs is the formulation of coated drug beads. Various coating materials were used for this purpose, such as ethyl cellulose and Eudragit ${ }^{\circledR}(5)$.

In the present study, cefaclor was used as a model drug. Cefaclor is a broad spectrum antibiotic belonging to the family of second generation cephalosporins. Cefaclor is well absorbed in the body, with a peak serum concentration occurring within 30-60 min, which is significantly reduced in the presence of food, with no change in the total amount of drug absorbed. Cefaclor is rapidly excreted in urine, with an approximate half-life of two hours (6). Because of its short half-life and large dose, cefaclor is considered a good candidate for sustained release dosage forms.

The aim of the present study was to develop a controlled release delivery system of cefaclor using coated alginate-chitosan beads. The effects of stirring time and the addition of a solubilizing agent on the encapsulation efficiency, bead size and the drug release profile were investigated. In addition, the antimicrobial activity of the coated beads of cefaclor was also evaluated.

\section{EXPERIMENTAL}

\section{Materials}

Cefaclor was obtained as a gift from Julphar Pharmaceutical Company (UAE). Chitosan (CTS) medium molecular mass, dihydrogen sodium orthophosphate, disodium hydrogen orthophosphate and phosphate buffer saline (PBS) tablets ( $\mathrm{pH} 7.4)$ were all purchased from Sigma-Aldrich (UK). Cellulose acetate phthalate (CAP), calcium chloride, ethyl cellulose (EC), and shellac (benzyl ethyl 2-methyl-4-(2-phenylethynyl)-1,4-dihydropyridine-3,5-dicarboxylate) were from $\mathrm{BDH}$ Chemicals (UK), $\beta$-cyclodextrin, sodium alginate (SA) and oleic acid (OA) sodium were from Hopkin and Williams (UK). All other chemicals were of analytical grade. Staphylococcus aureus (ATCC 25923) and Klebsiella pneumoniae (ATCC 15380) were obtained from the Ibn-Sina Center for drug quality control and analysis (Iraq).

\section{Preparation of cefaclor loaded beads}

Cefaclor loaded beads were prepared using two polymers, alginate and chitosan-alginate combination. Table I summarizes the composition of the prepared cefaclor bead formulations.

\section{Preparation of alginate beads}

Alginate beads (F1) were prepared according to the method described by Kikuchi (7). Two grams of cefaclor were dispersed in $7 \%(\mathrm{~m} / \mathrm{V})$ sodium alginate aqueous solution under shaking in a water bath at $40{ }^{\circ} \mathrm{C}$ until a homogeneous solution was obtained. 
B. K. A. Rasool and S. A. Fahmy: Development of coated beads for oral controlled delivery of cefaclor: In vitro evaluation, Acta Pharm. 63 (2013) 31-44.

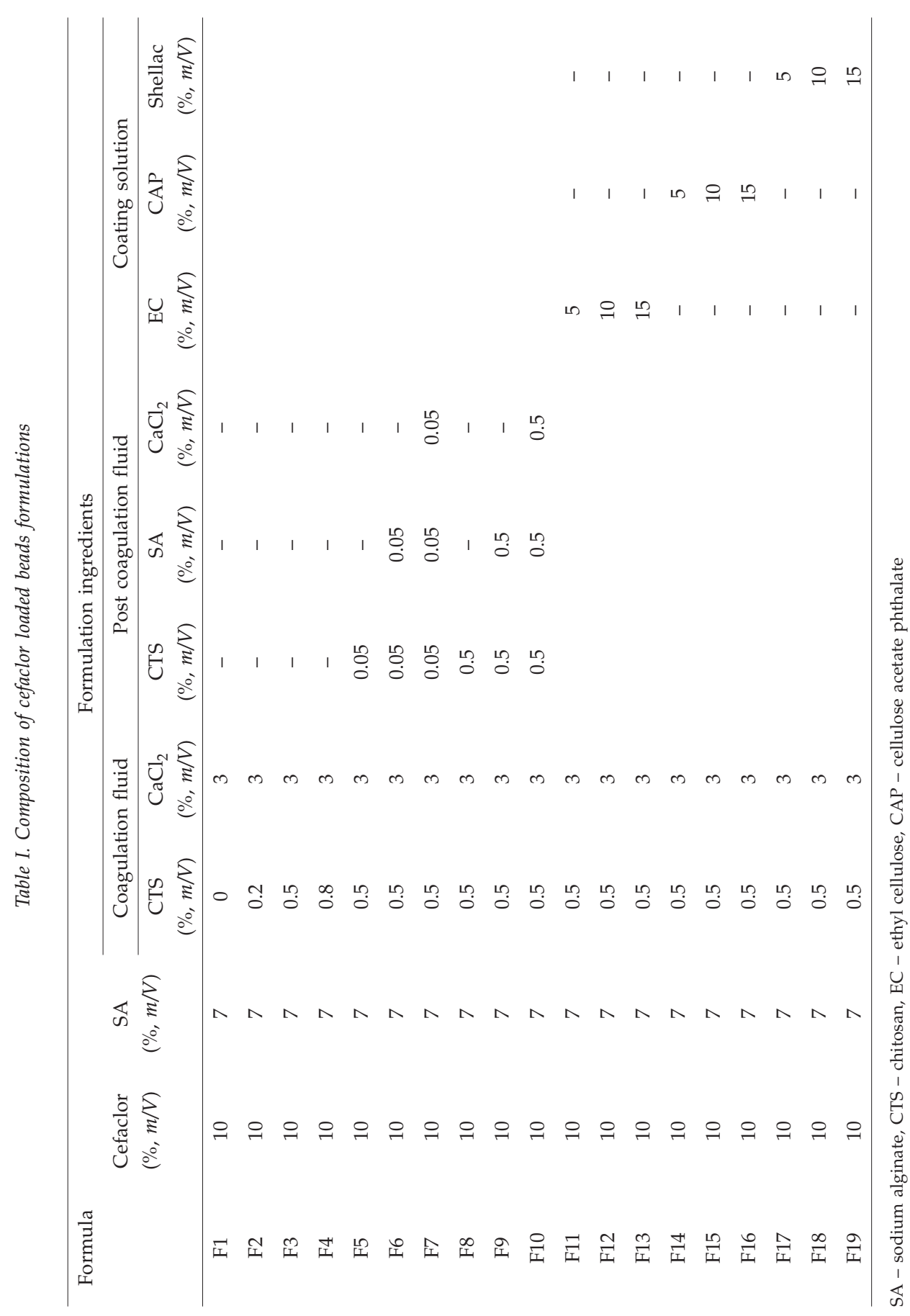


B. K. A. Rasool and S. A. Fahmy: Development of coated beads for oral controlled delivery of cefaclor: In vitro evaluation, Acta Pharm. 63 (2013) 31-44.

The $\mathrm{pH}$ was adjusted to 5.5 with a few drops of $0.1 \mathrm{~mol} \mathrm{~L}-1 \mathrm{NaOH}$. A volume of $20 \mathrm{~mL}$ of the solution was dropped through a 20 gauge syringe needle into $100 \mathrm{~mL}$ of a $3 \%(\mathrm{~m} / \mathrm{V})$ calcium chloride aqueous solution. The droplets were slowly stirred for $1 \mathrm{~h}$. Then, alginate beads were separated from the solution, washed several times with distilled water and dried at $60{ }^{\circ} \mathrm{C}$ for $12 \mathrm{~h}$.

\section{Preparation of chitosan-alginate beads}

Alginate-chitosan beads (F2-F4) were prepared by the ionotropic gelation method. Cefaclor was dispersed in a sodium alginate $7 \%(\mathrm{~m} / \mathrm{V})$ aqueous solution. The solution was dropped through a 20 gauge syringe needle into $100 \mathrm{~mL}$ of a $3 \%(\mathrm{~m} / \mathrm{V})$ of calcium chloride agitated solution ( $\mathrm{pH}$ adjusted to 5.5) containing different concentrations of chitosan $(0.2,0.5$ and $0.8 \%, m / V)$ already dissolved in $1 \%(V / V)$ acetic acid solution. Beads were left for $30 \mathrm{~min}$, and washed twice with distilled water and dried at $50{ }^{\circ} \mathrm{C}$ for $4 \mathrm{~h}$, and then at room temperature for 2 days for complete drying.

\section{Preparation of multilayer chitosan-alginate beads (F5-F10)}

Multilayer beads (F5) were prepared by placing the prepared cefaclor-chitosan alginate beads into $100 \mathrm{~mL}$ of the post coagulation solution, CTS solution $0.05 \%(\mathrm{~m} / \mathrm{V})$, for $30 \mathrm{~min}$ with stirring. F6 was prepared by placing the beads into $100 \mathrm{~mL}$ of $0.05 \%$ CTS and $100 \mathrm{~mL}$ of $0.05 \% \mathrm{SA}(\mathrm{m} / \mathrm{V})$ for $30 \mathrm{~min}$ with stirring. F7 was prepared by incubation of chitosan-alginate beads in $100 \mathrm{~mL}$ of $0.05 \%$ CTS and $100 \mathrm{~mL}$ of $0.05 \%$ SA for $30 \mathrm{~min}$, followed by $10 \mathrm{~min}$ stirring in $0.5 \%(\mathrm{~m} / \mathrm{V}) \mathrm{CaCl}_{2}$ aqueous solution. Indeed, $\mathrm{F} 8, \mathrm{~F} 9$ and F10 were prepared using the same former steps but the concentration of coagulation solutions (CTS, SA and $\mathrm{CaCl}_{2}$ ) was increased to $0.5 \%(\mathrm{~m} / \mathrm{V})$ (Table I). Chitosan-alginate multilayer beads were washed with distilled water and dried in an oven at $30{ }^{\circ} \mathrm{C}$ for $24 \mathrm{~h}$.

\section{Preparation of coated beads}

Cefaclor loaded chitosan-alginate beads were coated by the solvent evaporation technique. The beads were coated with ethyl cellulose (EC), cellulose acetate phthalate (CAP) or shellac. In brief, coating solutions were prepared by placing two grams of dried chitosan-alginate beads in $20 \mathrm{~mL}$ of each of the coating polymer solutions using different concentrations: 5, 10 and $15 \%(\mathrm{~m} / \mathrm{V})$. Formulation compositions (F11-F19) are shown in Table I. The coating polymeric solutions were prepared as follows. Ethyl cellulose solution was prepared by dissolving EC in chloroform while CAP and shellac were prepared by dissolving the polymer in a 1:1 isopropanol/acetone $(V / V)$ solution. The coating process was maintained at a stirring rate of $20 \mathrm{rpm}$ and temperature of $50{ }^{\circ} \mathrm{C}$ until complete evaporation of the solvent took place. Coated beads were left overnight at room temperature to ensure solvent evaporation.

\section{In vitro evaluation of the prepared beads}

The prepared formulations were evaluated for their morphological features, particle size, yield percent, entrapment efficiency and in vitro release of cefaclor from the prepared beads. 
B. K. A. Rasool and S. A. Fahmy: Development of coated beads for oral controlled delivery of cefaclor: In vitro evaluation, Acta Pharm. 63 (2013) 31-44.

\section{Morphological and particle size analysis}

An optical microscope (Eclipse TS 100, Nikon, Japan) was used to study the morphology and particle size of dried beads. The microscope eyepiece was fitted with a micrometer by means of which the particle size of the micro-beads could be determined. The particle size was measured as mean \pm SD of 100 examined beads.

\section{Entrapment efficiency}

Ten mg of dried loaded beads were pulverized and incubated in $10 \mathrm{~mL}$ phosphate buffer ( $\mathrm{pH} 7.4)$ at room temperature for $24 \mathrm{~h}$. The suspension was then centrifuged at $6000 \mathrm{rpm}$ for $30 \mathrm{~min}$. The supernatant was assayed for cefaclor using a UV spectrophotometer (Cintra 5, GBC Scientific equipment, Australia) at $\lambda_{\max }$ of $280 \mathrm{~nm}$. The supernatant from the empty beads was used as a blank.

In addition, the impact of some factors on the entrapment efficiency of cefaclor in chitosan-alginate beads (F5) was evaluated including the concentration of chitosan ( 0 , $0.2,0.5$ and $0.8 \%, \mathrm{~m} / \mathrm{V})$ and cefaclor $(5$ and $10 \% \mathrm{~m} / \mathrm{V})$, stirring time $(2,30,60$ and $120 \mathrm{~min})$ and additives' types and concentrations: $\mathrm{PEG}_{400} 1$ and $2 \%(V / V)$, oleic acid 1 and $2 \%(V / V)$ and $\beta$-cyclodextrin 1 and $2 \%(\mathrm{~m} / \mathrm{V})$. Different additives with different concentrations were added during bead preparation by adding the exact volume of the additive solution to the dispersion of cefaclor in the chitosan solution with stirring till a homogenous solution was obtained.

\section{In vitro release study}

The in vitro release study of the prepared formulae was carried out using a USP dissolution apparatus Type 1 (Copley, UK). The study was carried out by placing a weighed quantity of beads equivalent to $500 \mathrm{mg}$ cefaclor into hard gelatin capsules, in $900 \mathrm{~mL}$ of different dissolution media ensuring sink conditions. The dissolution media used in the study were $0.1 \mathrm{~mol} \mathrm{~L}^{-1} \mathrm{HCl}(\mathrm{pH}$ 1.2) for $2 \mathrm{~h}$, then acetate buffer $(\mathrm{pH} 4.6)$ for $1 \mathrm{~h}$, followed by phosphate buffer ( $\mathrm{pH}$ 6.8). The dissolution temperature was maintained at $37^{\circ} \mathrm{C}$ with a rotation rate of $100 \mathrm{rpm}$. Filtered samples were withdrawn at certain time intervals and replaced with an equal volume of fresh medium. The samples were analyzed for cefaclor content using a UV spectrophotometer adjusted to $280 \mathrm{~nm}$ (7).

\section{Kinetic analysis of in vitro release}

The in vitro drug release profiles of all formulae were analyzed by fitting the first $60 \%$ fraction of drug released to, the empirical equation proposed by Korsmeyer et al. (9):

$$
M_{\mathrm{t}} / M_{\infty}=k t^{\mathrm{n}}
$$

where $M_{t} / M_{\infty}$ is the fraction of drug released at time $t, k$ is the rate constant and $n$ is the release exponent. The exponential release constant $n$ was used to characterize the release mechanism. The rate constant $k$ and the diffusion exponent were calculated from the intercept and the slope of a logarithmic plot of F vs. $t$. 
B. K. A. Rasool and S. A. Fahmy: Development of coated beads for oral controlled delivery of cefaclor: In vitro evaluation, Acta Pharm. 63 (2013) 31-44.

\section{Microbiological study}

Antibiotic disc preparation. - Microbiological study of cefaclor released from the selected bead formulation was performed according to the standardized single disc method as described by Bauer et al. (10). Briefly, the standard antibiotic disc (15 $\mu \mathrm{g}$ per disc) was prepared by dissolving $1.5 \mathrm{mg}$ of cefaclor in $100 \mathrm{~mL}$ of phosphate buffer ( $\mathrm{pH}$ 7.4). One milliliter of this solution was placed on a previously prepared multi-filter paper disc and left to dry for $12 \mathrm{~h}$ at room temperature. Sample antibiotic discs were loaded with one milliliter of cefaclor solution released from F19 in phosphate buffer ( $\mathrm{pH} 7.4$ ) after 7 and 8 hours of dissolution. This concentration was measured against a previously prepared cefaclor calibration curve in phosphate buffer $\mathrm{pH} 7.4$ and found to be 15 and $20 \mu \mathrm{g}$ per disc, respectively.

\section{Antibiotic sensitivity test}

Antibiotic sensitivity test was carried out by transferring five colonies of each type of bacteria, Staphylococcus aureus and Klebsiella pneumoniae from the pure culture to $5 \mathrm{~mL}$ of a nutrient broth tube and incubated at $35^{\circ} \mathrm{C}$ for $2-5 \mathrm{~h}$ to produce a moderately cloudy suspension, which was standardized by dilution in turbidity standard barium chloride solution (TSB), visually equivalent to the McFarland standard 0.5 (standard turbidity prepared by adding $0.5 \mathrm{~mL}$ of $1 \%$ barium chloride solution to $99.5 \mathrm{~mL}$ of $1 \%$ sulphuric acid). A volume of $0.1 \mathrm{~mL}$ of this suspension was added to non-cultured nutrient agar, spread using a sterilized swab over the entire agar surface and incubated for $24 \mathrm{~h}$ at $37^{\circ} \mathrm{C}$. The prepared standard and sample antibiotic discs were placed at suitable distances on the cultured nutrient agar medium and incubated for $24 \mathrm{~h}$ at $37^{\circ} \mathrm{C}$ and the diameters of the produced inhibition zones were measured.

\section{In vivo convolution study}

Convolution was conducted to simulate the plasma concentration time curves for cefaclor from F13, F16, F19 and the uncoated bead formulations using spreadsheets. Although simulated data are not always close to real data, convolution is still a good tool to predict the time course of a drug in the body. Convolution was completed with the following assumptions: (i) drug elimination follow first-order elimination, (ii) no absorption phase is considered; drug is treated like a series of intravenous bolus injections with appropriate adjustment of bioavailability, (iii) absorption rate constant is greater than the dissolution rate constant, and (iv) the drug follows linear pharmacokinetics.

Simulated plasma concentration time profiles were compared to those of uncoated beads and the published data of plasma concentration time profiles for commercially available cefaclor formulations. The pharmacokinetic parameters (PK) of cefaclor were used as reported in literature to simulate cefaclor concentration. The following reported PK were used in the simulation: cefaclor bioavailability from immediate release formulations reported to be about $80 \%$, clearance $328.0 \pm 83.1 \mathrm{~mL} \mathrm{~min}^{-1}$ and elimination half-life of $0.6 \pm 0.1 \mathrm{~h}(11)$.

\section{Statistical analysis}

Results are expressed as mean \pm SD for triplicate samples. The results were statistically analyzed to test any significant differences among the groups by the one-way analysis of variance (ANOVA) using SPSS statistical analysis program version 16. 
B. K. A. Rasool and S. A. Fahmy: Development of coated beads for oral controlled delivery of cefaclor: In vitro evaluation, Acta Pharm. 63 (2013) 31-44.

\section{RESULTS AND DISCUSSION}

\section{Morphological features of the prepared beads}

Cefaclor loaded alginate beads (F1 to F4) using calcium chloride with or without chitosan were evaluated physically and found to be intact and spherical in shape. It was observed that the addition of chitosan to the coagulation solution produced beads with a smoother surface then that of alginate alone, as shown in Fig. 1.

Multilayer chitosan-alginate beads (F5-F10) were found to be intact and compact. Use of chitosan in the preparation of alginate beads resulted in spherical beads with a smooth surface. This may be attributed to the fact that chitosan bounds electrostatically to the alginate polymer molecules present on the surface of the beads. The beads that were additionally treated with sodium alginate (F6 and F9) were found to be clumpy after washing. An extra treatment with $\mathrm{CaCl}_{2}(0.5 \%, \mathrm{~m} / \mathrm{V})$ also resulted in smooth and spherical beads (F7 and F10) where $\mathrm{Ca}^{2+}$ ions were cross-linked with the outer most alginate layer of the beads to yield a complex structure (12).

\section{Bead yield and bed size}

The yield of prepared beads was almost 80 to $90 \%$ for all the formulations. It was noticed that the bead yield was slightly lower (70-75\%) in the formulations where chitosan concentration was low $(0.05 \%, \mathrm{~m} / \mathrm{V})$.

Mean sizes of the prepared beads of different formulations are shown in Table II. The mean particle size of the prepared beads was in the following order: chitosan-alginate beads $(1.05-1.37 \mathrm{~mm})<$ chitosan-alginate multilayer beads $(1.34-1.65 \mathrm{~mm})<$ chitosan-alginate coated beads $(1.68-2.08 \mathrm{~mm})$. There was no significant difference in particle size between multilayer beads (F5, F6 and F7) and chitosan-alginate beads (F3 and F4). However, there was a significant difference $(p<0.05)$ in particle size between the multilayer bead formulae (F8, F9 and F10) and the chitosan-alginate bead formulae (F1, F2, F3 and F4). On the other hand, coated beads were significantly larger than chitosan-alginate single and multilayer beads.

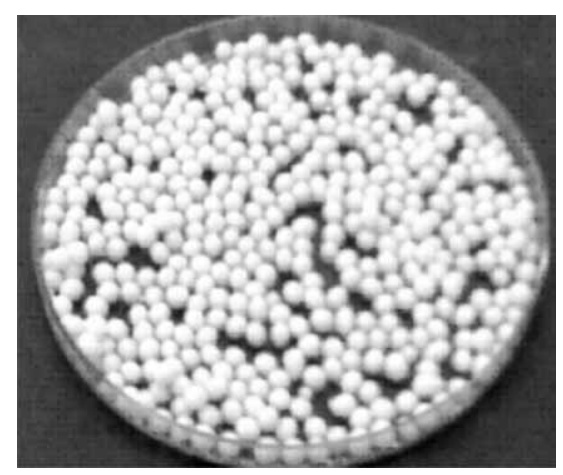

Fig. 1. Microscope picture of cefaclor loaded chitosan-alginate beads. 
B. K. A. Rasool and S. A. Fahmy: Development of coated beads for oral controlled delivery of cefaclor: In vitro evaluation, Acta Pharm. 63 (2013) 31-44.

Table II. Factors affecting cefaclor entrapment efficiency in chitosan-alginate beads

\begin{tabular}{|c|c|c|c|c|c|c|}
\hline Factor & & & Entrapment & ficiency $(\%)$ & & \\
\hline Chitosan & $0(\mathrm{~F} 1)$ & $0.2(\mathrm{~F} 2)$ & & & & 4) \\
\hline$(\%, m / V)$ & $18.9 \pm 2.5$ & $29.1 \pm 4.1$ & 49. & 6.8 & 49. & 9.6 \\
\hline Stirring time & 2 & 30 & & & & \\
\hline$(\min )$ & $63.2 \pm 7.9$ & $48.9 \pm 6.86$ & 34. & 3.9 & 26. & 5.4 \\
\hline Additive & PEG 40 & $(\% V / V)$ & Oleic ac & $(\%, V / V)$ & $\beta-$ Cyclode & n $(\%, m / V)$ \\
\hline & 1 & 2 & 1 & 2 & 1 & 2 \\
\hline & $67.6 \pm 10.6$ & $72.7 \pm 12.7$ & $63.7 \pm 8.3$ & $64.5 \pm 9.3$ & $63.9 \pm 9.6$ & $64.2 \pm 10.5$ \\
\hline Cefaclor & 5 & 10 & & & & \\
\hline$(\%, m / m)$ & $57.8 \pm 7.6$ & $72.7 \pm 11.4$ & & & & \\
\hline
\end{tabular}

${ }^{\mathrm{a}}$ Mean $\pm \mathrm{SD}, n=3$.

\section{Entrapment efficiency}

The effect of certain factors on the entrapment efficiency was studied on a selected cefaclor formulation (F5) (Table III). Increasing chitosan concentration in the coagulation solution was found to lead to significant increase in the drug entrapment efficiency with $p<0.05$. Almost the same entrapment efficiency was obtained when chitosan concentration increased from 0.5 to $0.8 \%$ in formulae F3 and F4, respectively. Formula F1, with no chitosan, showed the lowest drug entrapment efficiency $(18.9 \%)$. This may be attributed to gelation of the polymer at $\mathrm{pH} 4.5$ where interaction between the carboxylic groups of the sodium alginate molecules and the protonated amine groups in chitosan results in the formation of a firm netlike complex of chitosan-alginate that tightly holds the drug inside the beads (13). However, the absence of chitosan in formula F1 resulted in inadequate cross-linking of polymer molecules and led to the formation of large pore size which permitted drug diffusion and consequently resulted in very low entrapment efficacy of cefaclor.

Increasing the stirring time during bead preparation resulted in a decrease in cefaclor entrapment efficiency, which may be due to the increased possibility of drug leaching outside the beads.

However, increasing the concentration of cefaclor from 5 to $10 \%(\mathrm{~m} / \mathrm{V})$ resulted in better drug entrapment in the beads.

The addition of additives during bead preparation resulted in good entrapment efficiency compared to beads with no additive. PEG 400 (2\%) led to a significant increase $(p<0.05)$ in the entrapment efficacy compared to beads prepared with the addition of $1 \%$ PEG 400. This may be attributed to the fact that PEG 400 is a solubilizing agent and freely soluble in water; it therefore enhanced the solubility of cefaclor in sodium alginate solution and thereby improved the entrapment efficiency of the drug inside the beads.

However, increasing the concentration of oleic acid and $\beta$-cyclodextrin from 1 to $2 \%$ $(V / V)$ did not significantly increase drug entrapment efficiency. 
B. K. A. Rasool and S. A. Fahmy: Development of coated beads for oral controlled delivery of cefaclor: In vitro evaluation, Acta Pharm. 63 (2013) 31-44.

Table III. Particle size and kinetic parameters of cefaclor loaded chitosan-alginate beads

\begin{tabular}{cccccc}
\hline Formula & $\begin{array}{c}\text { Particle size } \\
(\mathrm{mm})^{\mathrm{a}}\end{array}$ & $\begin{array}{c}\text { ln } k \\
\left(\%, \mathrm{~min}^{-1}\right)\end{array}$ & $n$ & $R^{2}$ & Diffusion model \\
\hline F1 & $1.05 \pm 0.07$ & 4.321 & 0.069 & 0.976 & Fickian diffusion \\
F2 & $1.18 \pm 0.13$ & 4.132 & 0.125 & 0.977 & Fickian diffusion \\
F3 & $1.33 \pm 0.09$ & 4.123 & 0.093 & 0.984 & Fickian diffusion \\
F4 & $1.37 \pm 0.13$ & 4.057 & 0.105 & 0.977 & Fickian diffusion \\
F5 & $1.34 \pm 0.12$ & 4.105 & 0.0942 & 0.937 & Fickian diffusion \\
F6 & $1.36 \pm 0.21$ & 4.026 & 0.111 & 0.972 & Fickian diffusion \\
F7 & $1.38 \pm 0.27$ & 4.049 & 0.102 & 0.976 & Fickian diffusion \\
F8 & $1.62 \pm 0.18$ & 3.916 & 0.121 & 0.914 & Fickian diffusion \\
F9 & $1.65 \pm 0.23$ & 3.431 & 0.208 & 0.891 & Fickian diffusion \\
F10 & $1.65 \pm 0.15$ & 3.278 & 0.228 & 0.901 & Fickian diffusion \\
F11 & $1.95 \pm 0.31$ & 1.459 & 0.561 & 0.94 & Anomalous diffusion \\
F12 & $2.04 \pm 0.28$ & 1.442 & 0.528 & 0.963 & Anomalous diffusion \\
F13 & $2.06 \pm 0.38$ & 1.144 & 0.574 & 0.972 & Anomalous diffusion \\
F14 & $1.68 \pm 0.22$ & 1.87 & 0.498 & 0.972 & Fickian diffusion \\
F15 & $1.81 \pm 0.36$ & 1.879 & 0.497 & 0.956 & Fickian diffusion \\
F16 & $1.85 \pm 0.27$ & 1.762 & 0.518 & 0.972 & Anomalous diffusion \\
F17 & $1.90 \pm 0.34$ & 2.119 & 0.445 & 0.973 & Fickian diffusion \\
F18 & $2.06 \pm 0.37$ & 2.134 & 0.369 & 0.971 & Fickian diffusion \\
F19 & $2.08 \pm 0.31$ & 2.225 & 0.365 & 0.972 & Fickian diffusion \\
\hline
\end{tabular}

a Mean $\pm \mathrm{SD}, n=3$.

\section{In vitro drug release}

The release profile of cefaclor from bead formulations F1-F4 showed a rapid burst effect during the first 30 minutes of dissolution (Fig. 2a). This rapid release may be explained as follows. First, when chitosan-alginate loaded beads were placed at a low $\mathrm{pH}$ (1.2), the negatively charged carboxylate groups of sodium alginate began to protonate to form uncharged $-\mathrm{COOH}$ groups. This reduced the degree of cross linking due to decreased electrostatic interactions among the alginate and chitosan chains within the beads, resulting in increasing fluids uptake and swelling of the beads (12). In addition, cefaclor as a small, hydrophilic molecules at $\mathrm{pH} 1.2$ is highly ionized, resulting in an increase in drug solubility; hence it diffuses out easily.

Release profiles of the chitosan-alginate multilayer beads (F5-F10) are shown in Fig. $2 \mathrm{~b}$. Increasing chitosan concentration in the multilayered bead formulation F8 resulted in a decrease in drug release compared to that of formulation F5. In both formulae, deprotonation of the $\mathrm{NH}_{4}{ }^{+}$group in the outermost chitosan layer to $\mathrm{NH}_{2}$ groups occurred upon placing the beads in a simulated intestinal fluid, which can form a diffusion barrier for $\mathrm{Na}^{+} / \mathrm{Ca}^{2+}$ ion exchange among $\mathrm{Ca}^{2+}$ ions in the bulk of the beads and $\mathrm{Na}^{+}$ 
B. K. A. Rasool and S. A. Fahmy: Development of coated beads for oral controlled delivery of cefaclor: In vitro evaluation, Acta Pharm. 63 (2013) 31-44.

a)

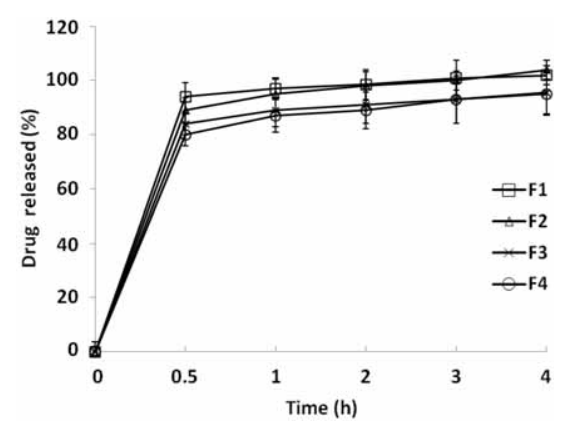

c)

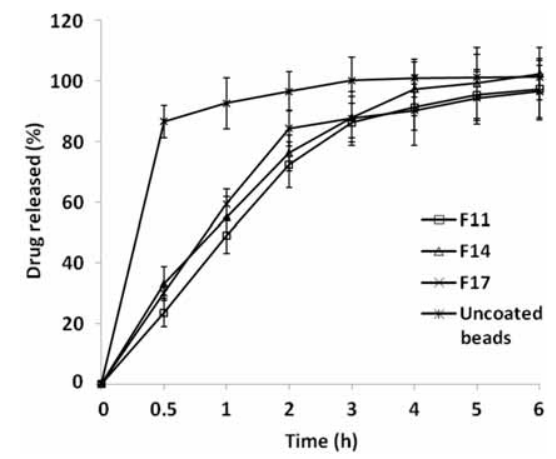

e)

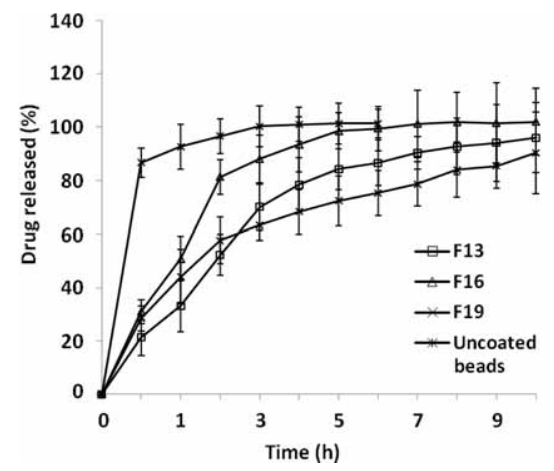

b)

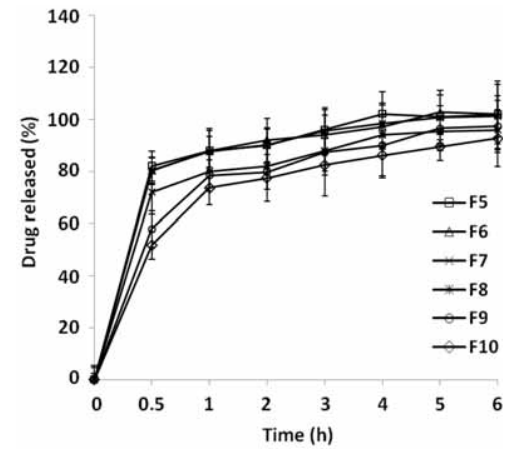

d)

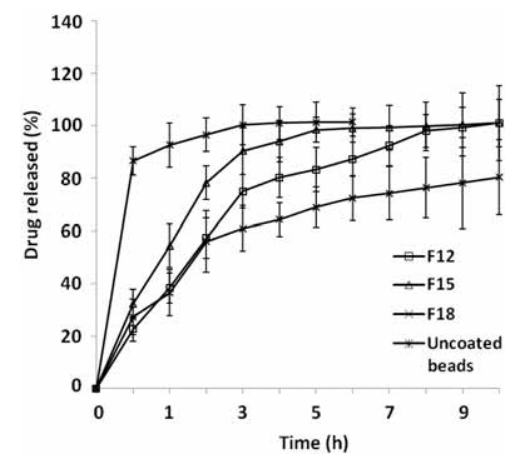

Fig. 2. Cefaclor release profile from chitosan-alginate beads: a) formulated with different chitosan concentrations; b) multilayered beads in the post granulation fluid; c) coated with $5 \%(\mathrm{~m} / \mathrm{V})$ ethyl cellulose (F11), CAP (F14) and shellac (F17); d) coated with $10 \%(\mathrm{~m} / \mathrm{V})$ ethyl cellulose (F12), CAP (F15) and shellac (F18); e) coated with $15 \%(\mathrm{~m} / \mathrm{V})$ ethyl cellulose (F13), CAP (F16) and shellac (F19).

ions in the simulated intestinal fluid. By increasing chitosan concentration in the post coagulation solution (F8), the barrier hindrance also increased and consequently the bead disintegration process was delayed (14).

It was observed that there was a significant difference in the release profile of $\mathrm{F} 6$ and F9 after the first 30 minutes of dissolution. After 30 minutes, F6 released $81 \%$ of cefaclor compared to $57 \%$ released from formula F9. The outermost layer of both F6 
and F9 was sodium alginate. In simulated gastric fluid ( $\mathrm{pH} 1.2$ ), the negative - $\mathrm{COO}^{-}$ group of sodium alginate got protonated and acted as a diffusion barrier for the release of the drug. Increasing the thickness of this layer will result in increasing the barrier of drug diffusion and thereby more retardation in drug release. Upon treating the beads with simulated intestinal fluids, the alginic acid will begin to dissolve, leaving chitosan forming the outermost layer. Chitosan will act as a diffusion barrier for $\mathrm{Na}^{+} / \mathrm{Ca}^{2+}$ ion exchange, which will result in delayed drug release. This explain why F9 showed more delayed drug release than F6 (15).

Drug release from F10 formulations was retarded, with only $65.6 \%$ of the drug release after 30 minutes of dissolution compared to $80.3 \%$ from formulation F7. This may be attributed to the addition of calcium chloride where the $\mathrm{Ca}^{2+}$ ions act as a hardening agent for the outer layer of beads. Therefore, a rigid layer was produced that delayed diffusion and drug release.

The overall results of release profiles for chitosan-alginate and chitosan-alginate multilayer beads demonstrate a burst effect during the first hour of dissolution and no prolonged release of cefaclor was obtained from the prepared formulations (F5-F10).

Chitosan alginate beads coated with $5 \%(\mathrm{~m} / \mathrm{V})$ ethyl cellulose (F11), $5 \%$ CAP (F14) and $5 \%(\mathrm{~m} / \mathrm{V})$ shellac (F17) showed a delay in drug release from the prepared formulations of $23.7,33.2$ and $30.2 \%$, respectively, after 30 minutes, compared to $86.6 \%$ of drug released from the uncoated formulations (Fig. 2c).

Good sustained drug release was obtained from chitosan-alginate coated formulations using $10 \%(\mathrm{~m} / \mathrm{V})$ of polymeric solutions, especially with $10 \%(\mathrm{~m} / \mathrm{V})$ of EC $(\mathrm{F} 12)$ and shellac (F18) formulations. For F15 bead formulation coated with $10 \%(\mathrm{~m} / \mathrm{V})$ CAP, drug release was faster with more than $90 \%$ drug released after $4 \mathrm{~h}$, which was similar in pattern to the drug released from $5 \%(\mathrm{~m} / \mathrm{V})$ CAP coated bead formulation. Data are shown in Fig. 2d.

Increasing the concentration of the coating polymeric solution to $15 \%(\mathrm{~m} / \mathrm{V})(\mathrm{F} 13$, F16 and F19) showed release patterns dissimilar to those obtained from the $10 \%(\mathrm{~m} / \mathrm{V})$ coated formulations. There was a significant difference $(p<0.05)$ in cefaclor release from chitosan-alginate beads coated with $15 \%(\mathrm{~m} / \mathrm{V})$ of polymeric materials compared to $10 \%$ of the coating polymer (Fig. 2e). F19 (shellac $15 \%$ coated beads) was considered the most successful formula for controlled cefaclor release and was selected for further study.

Differences in the release profile among coated chitosan-alginate bead formulations using different polymers are attributed to the differences in solubility of the polymeric materials used, where CAP solubilizes at $\mathrm{pH} 5$ or more while ethyl cellulose remains intact throughout the GIT and shellac often dissolves too slowly in the intestinal fluid (16). As a result, there is faster solubilization of CAP than EC and shellac while the beads passing through the intestinal fluid $(\mathrm{pH} 4.6$ and 6.8) result in faster drug solubilization and diffusion into the intestinal fluid.

\section{Kinetic analysis of in vitro drug release}

Fractions of the drug released from all developed formulae were fitted using an empirical equation. Straight lines were obtained from plotting $\ln M_{t} / M_{\infty} v s$. $\ln t$ and the kinetic parameter $(n)$ was calculated, with the correlation coefficients $(R)$ and $95 \%$ confidence intervals for all formulations (Table II). The values of $n$ obtained for most 
B. K. A. Rasool and S. A. Fahmy: Development of coated beads for oral controlled delivery of cefaclor: In vitro evaluation, Acta Pharm. 63 (2013) 31-44.

Table IV. Inhibition zone diameter $(\mathrm{mm})$ of cefaclor discs incubated in two culture media

\begin{tabular}{|c|c|c|}
\hline $\operatorname{Disc}^{a}$ & Staphylococcus aureus ${ }^{\mathrm{b}}$ & Klebsiella pneumoniae ${ }^{\mathrm{b}}$ \\
\hline Cefaclor & $24 \pm 1$ & $15 \pm 0$ \\
\hline F19 formula & $33 \pm 0$ & $17 \pm 1$ \\
\hline F19 formula & $35 \pm 1$ & $19 \pm 0$ \\
\hline
\end{tabular}

a $15 \mu \mathrm{g}$ per disc

b Mean \pm SD, $n=3$.

of the formulations are less than 0.5 , which suggests drug release from the prepared formulations via Fickian release mechanism. However, the values of $n$ for the drug released from CAP coated chitosan-alginate beads were greater than 0.5 , indicating anomalous drug release from the beads by both diffusion and polymer erosion mechanisms.

\section{Microbiological assay}

The amount of cefaclor released from F19 after 7 and $8 \mathrm{~h}$ was $15 \mu \mathrm{g}$ and $20 \mu \mathrm{g}$, respectively. The growth inhibition zones of both Staphylococcus aureus and Klebsiella pneumoniae were measured and significantly higher antibacterial activity $(p<0.05)$ was observed for formula F19 compared to the standard antibiotic disc (Table IV). These results demonstrate the efficiency of the new formulation (F19) as a sustained antimicrobial oral dosage form.

\section{Convolution}

Simulation was done to predict the drug plasma concentration from the various formulae administered in vivo. Results are presented in Fig. 3. Simulated data showed that

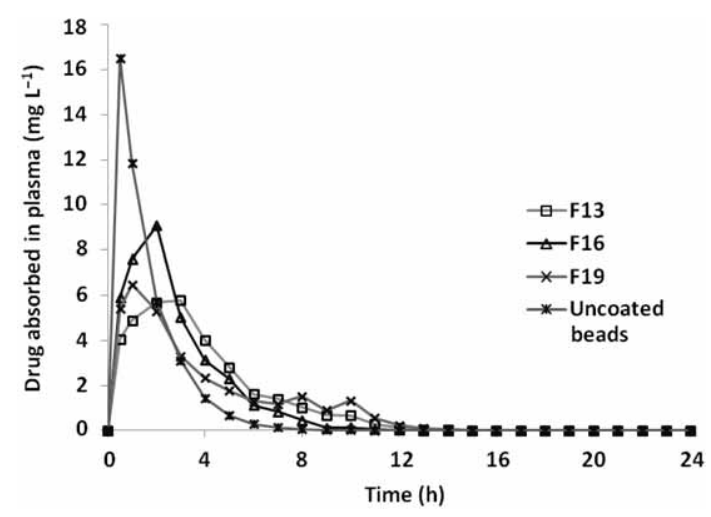

Fig. 3. Simulated Cefaclor plasma concentration time profiles after the administration of $500 \mathrm{mg}$ per body mass oral dose of selected beads formulations, F13, F16, F19 and the uncoated beads formulations. 
B. K. A. Rasool and S. A. Fahmy: Development of coated beads for oral controlled delivery of cefaclor: In vitro evaluation, Acta Pharm. 63 (2013) 31-44.

uncoated beads produced the highest concentration $\left(\gamma_{\max }\right)$ in the blood stream, with a concentration of $16.5 \mathrm{mg} \mathrm{L}^{-1}$ reached within 30 minutes, and cefaclor plasma level was lower than $0.1 \mathrm{mg} \mathrm{L}^{-1}$ after $8 \mathrm{~h}$. $\gamma_{\max }$ obtained from the uncoated bead formulations was comparable to that reported for cefaclor immediate release dosage form at a dose of $500 \mathrm{mg}$ per body mass $\left(17.3 \pm 3.6 \mathrm{mg} \mathrm{L}^{-1}\right)$. For formula F13, at the simulated drug plasma profile was maximum $\left(5.77 \mathrm{mg} \mathrm{L}^{-1}\right)$ after $3 \mathrm{~h}$ and cefaclor concentration was less than $0.1 \mathrm{mg} \mathrm{L}^{-1}$ after $12 \mathrm{~h}$, which proves the sustained release action of cefaclor from coated beads formulations. For F16 and F19, the maximum concentration of $9.07 \mathrm{mg} \mathrm{L}^{-1}$ was reached after $2 \mathrm{~h}$ and $6.5 \mathrm{mg} \mathrm{L}^{-1}$ after one hour, respectively. Cefaclor concentration reached less than $0.1 \mathrm{mg} \mathrm{L} \mathrm{L}^{-1}$ after $11 \mathrm{~h}$ and $13 \mathrm{~h}$ from F16 and F19 formulae, respectively.

The simulated plasma profile for the selected coated formulations showed the superiority of F19 and F13 in sustaining drug release for more than $12 \mathrm{~h}$.

\section{CONCLUSIONS}

Cefaclor loaded chitosan-alginate coated beads were developed successfully for modified oral delivery of cefaclor. The desired entrapment efficiency of cefaclor in the prepared beads was optimized by adjusting different parameters such as polymer type concentration of the solubilizing agent and stirring time.

The best in vitro sustained release pattern of cefaclor was obtained from bead formulations coated with shellac $15 \%(\mathrm{~m} / \mathrm{V})$ (F19). In addition, the microbiological activities demonstrated that F19 is effective against Staphylococcus aureus and Klebsiella pneumoniae. There was a significant difference in its activity against $(p<0.05)$ Staphylococcus aureus and Klebsiella pneumoniae compared to the standard. Convolution of the in vitro dissolution data showed a good sustained release action from both the F19 and F13 formulae over $12 \mathrm{~h}$.

Acknowledgements. - The authors thank Julphar Pharmaceutical Company (Ras Al Khaima, UAE) for providing cefaclor as well as the Ibn-Sina Center for drug quality control and analysis, Baghdad, Iraq, for their support in the microbiology assay.

\section{REFERENCES}

1. A. Kakkar, Characterization of ibuprofen loaded microcapsules prepared by ionotropic gelation, Indian J. Pharm. Sci. 57 (1995) 56-60.

2. L. Xing, C. Dawei, X. Liping and Z. Rongqing, Oral colon-specific drug delivery for bee venom peptide: development of a coated calcium alginate gel beads-entrapped liposome, J. Control. Release 93 (2003) 293-300; DOI: 10.1016/j.jconrel.2003.08.019.

3. K. Yao, T. Peng and Y. He, Swelling kinetics and release characteristics of cross linked chitosan-polyether polymer network (sem: IPN) hydrogels, J. Polym. Sci. A 32 (1994) 1213-1219.

4. A. Sezer and J. Akbûga, Release characteristics of chitosan treated alginate beads ii. Sustained release of a low molecular weight drug from chitosan treated alginate beads, J. Microencaps, 16 (1999) 687-696; DOI: 10.1080/026520499288636. 
B. K. A. Rasool and S. A. Fahmy: Development of coated beads for oral controlled delivery of cefaclor: In vitro evaluation, Acta Pharm. 63 (2013) 31-44.

5. N. Pearnchob and R. Bodmeier, Dry polymer powder coating and comparison with conventional liquid-based coatings for Eudragit RS, ethylcellulose and shellac, Eur. J. Pharm. Biopharm. 56 (2003) 363-369; DOI: 10.1016/S0939-6411(03)00121-8.

6. R. Wise, The pharmacokinetics of the oral cephalosporins-a review, Antimicrob. Agents Chemother. 26 (1990) 13-20.

7. A. Kikuchi, M. Kawabuchi, A. Watanabe, M. Sugihara, Y. Sakurai and T. Okano, Effect of Ca-alginate gel dissolution on release of dextran with different molecular weights, J. Control. Release 58 (1999) 21-28; DOI: 10.1016/S0168-3659(98)00141-2.

8. United States Pharmacopeia 30, National Formulary 25, Vol 2, USP Convention, Rockville (MD) 2007, pp. 277-284.

9. W. Korsmeyer, R. Gurny, E. Doelker, P. Buri and N. Peppas, Mechanisms of solute release from porous hydrophilic polymers, Int. J. Pharm. 15 (1983) 25-35; DOI: 10.1016/0378-5173(83)90064-9.

10. A. Bauer, M. Kirby, C. Sherris and M. Turck, Antibiotic susceptibility testing by standardized single disk method, Am. J. Clin. Pathol. 45 (1966) 493-496.

11. R. Barbhaiya, U. Shukla, C. Gleason, W. Shyu, R. Wilber and K. Pittman, Comparison of cefprozil and cefaclor pharmacokinetics and tissue penetration, Antimicrob. Agents Chemother. 34 (1990) 1204-1209.

12. S. Bajpai and R. Tankhiwale, Investigation of water uptake behavior and stability of calcium alginate/chitosan bipolymeric beads: Part-1, React. Funct. Polym. 66 (2006) 645-658; DOI: 10.1016/ j.reactfunctpolym.2005.10.017.

13. D. Bhopatkar, A. Anal and W. Stevens, Ionotropic alginate beads for controlled intestinal protein delivery: effect of chitosan and barium counter-ions on entrapment and release, J. Microencaps. 22 (2005) 91-100; DOI: 10.1080/02652040400026434.

14. L. Whithead, H. Collet and T. Fell, Amoxycillin release from a floating dosage form based on alginate, Int. J. Pharm. 210 (2000) 45-49; DOI: 10.1016/S0378-5173(00)00567-6.

15. K. Chourasia and K. Jain, Pharmaceutical approaches to colon targeted drug delivery system, J. Pharm. Pharm. Sci. 6 (2003) 33-66.

16. N. Pearnchob, A. Daskevsky and R. Bodmeir, Improvement in the disintegration of shellac-coated soft gelatin capsules in simulated intestinal fluid, J. Control. Release 94 (2004) 313-321; DOI: 10.1016/ j.jconrel.2003.10.004. 